\title{
A Computer Aided Strategy for More Sustainable Products
}

\author{
Davide Russo \\ Industrial Engineering Department, University of Bergamo, \\ Viale Marconi 5, 24044 Dalmine(BG), Italy \\ davide.russo@unibg.it
}

\begin{abstract}
This article presents a methodological study on the potential use of structural optimization strategies for eco-design to support engineers during the design process of green products. The structure of the traditional process of ecodesign now depends on the personal skills of the designer and his ability to integrate in the traditional design process the requirements of eco-sustainability. The objective of this study was to address guidance on integrating environmental aspects into a computer aided inventing product design and development extending the functionality of the traditional design process to green product design. The proposal arises combining eco-inventive principles with the design approach based on structural optimization tools so creating a knowledge-rich CAE process linking CAD, FEM, Optimization tools and LCA based tool. CAI tool is used to generate unconventional geometries and to trace the best promising direction of intervention. LCA integration allows the user in real-time to measure the environmental impact of his design choice calculated as a variation on the main indexes of environmental sustainability. A case study about the eco improvement of a steering plate for trial motorcycles is presented and discussed with the aim to introduce potential benefits of such an inventive eco design approach.
\end{abstract}

Keywords: Eco-design, Inventive Design, Structural Optimization, CAI, Eco guidelines, TRIZ.

\section{Introduction}

In former times, engineers were only concerned about designing a product that met its objectives. Little attention was given to the future availability of natural resources. Current design trend is changing. The product not only has to fulfil its objectives/functional performances, but it also has to fulfil market needs and ensure minimal material and/or energy wastage. To make innovation means to begin a process in order to create value modifying a set of the system variables [19]. To achieve this goal, many tools have been conceived in order to identify where it is more useful to intervene, and how to improve current products and processes. To order them, Knight [1] grouped these tools in 3 categories:

1. Guidelines defined as providing broad support, with little detail, but applicable either across the whole product development process and lifecycle, covering a 
significant area (e.g. design for recycling; design for disassembly; design for lifetime optimization. See for example [1-4]).

2. Checklists defined as providing in-depth, but narrow, application at selected stages of the product development process or lifecycle (see for example [5-7]).

3. Analytical tools defined as providing detailed and/or systematic analysis at specific stages of either the product development process or lifecycle, e.g. eco-indicators; environmental effect analysis; environmental impact assessment; life cycle assessment; material, energy and toxicity ('MET') matrix; life cycle cost analysis (see for example [8-13]).

Most of them can be considered as excellent tools, they are easy to use, well organized, and time-efficient. However, they do not provide a mechanism to prioritize the environmental requirements and deploy them into the product development process [14]. The most structured and widespread tool for assessment is certainly Life Cycle Assessment (LCA); it is used to measure, evaluate and report the resource requirements (raw materials and energy) and environmental emissions related to a specific system. LCA has a key strength in its quantitative approach, but this technique requires a huge investment in time and effort, and it is "not useful in the design process. Companies cannot delay their design process to wait for the results." [15]. Unfortunately, LCA approach is too complex and not user friendly [16], and actually doesn't provide any suggestions about how to change design of current product.

Russo et al. [17], positioned a large selection of these tools and methods according to different criterions of classification, i.e. eco-assessment toward eco-innovation.

The capability of performing both assessment and improvement using a unique method could significantly increase the effectiveness of the design process and the robustness of results achieved, but at the moment there is not a winning paradigm to do that.

However, a complete CAI tool should provide suitable ideas according to an assessment phase comprising both performance and environmental requirements. This work put the methodological basis for a CAI concept integrating environmental requirements into product design and development. It combines environmental guidelines, with abridged LCA Software and virtual modeling based on CAD and Structural optimization SW.

An overview of simplified LCA and current tools for environmental requirements assessment/improuvement integration is proposed in order to limit time and costs of the traditional LCA[18]. A different approach is proposed by Darmstadt University [25], they decided to work in a CAD environment more congenial for the designer and generate results by the assessment phase directly in such environment. The promising developments offered by this approach will be analyzed and criticized in section 2.

The overall framework of this work will be presented in the following sections together with an exemplary case study. 


\section{The Integration of Computer Aided Design for the Sustainable Design}

Eco-design [21-22], or green-design, consists in a set of coordinated activities intended to develop products and processes with less environmental impact. The application of eco-design involves a particular framework for considering environmental issues, the application of relevant analysis and synthesis methods, and a challenge to traditional procedures for design and manufacturing. The designer is involved not only in the production and use phase, but also in the entire product life cycle with pre-manufacturing (raw material and processing), end of life and transports.

Four levels of eco-design can be defined as follows:

- Level 1- product improvement: this is a progressive and incremental improvement of the product, a re-styling of the product; for example, it can consist of decreasing the use of materials or replacing one type of fastener by another;

- Level 2- product redesign: a new product is redesigned on the basis of an existing product;

- Level 3- new product concept definition: this is an innovation rupture as technical functions to fulfill product functionality are different.

- Level 4- new production system definition: this occurs when innovation in the productive system is necessary.

In this work a proposal for an innovative framework, especially conceived for levels 1 and 2 of eco-design, is presented.

The suggested methodology is focused on redesigning sustainable products (solids and not fluids), owning at the first and second level of the previous classification. The reason of this limitation is due to the framework adopted to manage such a design process: CAD structure.

The most important tools in the traditional design process are $\mathrm{CAD}$ and $\mathrm{CAE}$ software. However, current $\mathrm{CAD}$ tools help engineers to design a product that meets the functional and structural constraints but they are not able to manage environmental constraints. Only few applications of CAD for eco-design have been identified in literature, and in most cases they are promoted by academic research groups except for Solidworks 2010 that provides a specific module for sustainability.

Although the introduction of eco-requirements in CAD environment is so poor, the structure of the information offers many advantages:

- Product (assembly) is already organized by separated parts allowing an arrangement of results by components.

- Information about material, transport and manufacture process, cost, etc. can be easily associated to each part, so involving other life cycle phases traditionally excluded in a standard design approach.

- Intuitively usable.

- Automatic calculation of masses and volumes by subordinate components or material density. 
- Material and item statistics can be easily implemented.

- Easy back tracing of ecological hotspots through the product structure.

The aim of the CAD base tool for Eco-design [23] is to measure variations on environmental impact indexes not at the end but during all the design activity, and so monitoring on time any change onto the product and at the same time allowing to trace the best promising direction of intervention.

EcologiCAD [25], developed in 2004, is probably the first attempt to undertake ecological Life Cycle Assessments during product development. The used structures are similar to the product structures in 3D-CAD systems and doesn't offer guidelines and not even list of materials.

Another interesting attempt is represented by ECOCAD [26], a tool that interfaces to a large number of other instruments, including:

(1) CAD platform with environmental information Product Data Management (PDM), Product Life-Cycle Management (PLM) and Data Base (DB), containing information for the design of mechanical components such as materials, production processes, volumes, geometries.

(2) Platform CAD, FEM and CAM used to create and manage geometric /mathematical information of the components.

(3) LCA and green Guidelines from eco-design pilot [27].

The advantages of this approach is that geometrical information and process are automatically extracted from CAD as volume, surface, and features (holes, bending sheet metal, welding). User has to set materials, connection systems between parts, and processes that are not directly managed by the CAD.

Although the approach framework is very promising, the analysis results are not very reliable, mainly due to two reasons:

- the difficulty of obtaining accurate, completed and updated information DB;

- the lack of an effective integration of design suggestions often representing common sense ("we do it anyway"), but lack specificity. So where they are already inherent in the design process they need recognition and development [16].

Finally, for completeness, an example of integration between structural optimization tools and design for disassembly [28] proposed by Willems is mentioned.

In this work CAD and structural optimization tools are currently used to offer to the user important suggestions to redesign the product.

\section{Research Approach}

A complete work of integration between the eco-improvement suggestions and an aLCA software has been developed by Russo et al. [21] during the European project "Recycling and Resource Efficiency driving innovation in European Manufacturing SMEs" (REMAKE project [29]) in 2010.

The aim of this method was to suggest a new way to perform the assessment phase, based on the identification of the hotspots on which to intervene. Such hotspots are identified by specific ideality indicators and information about resources already employed by the company. 


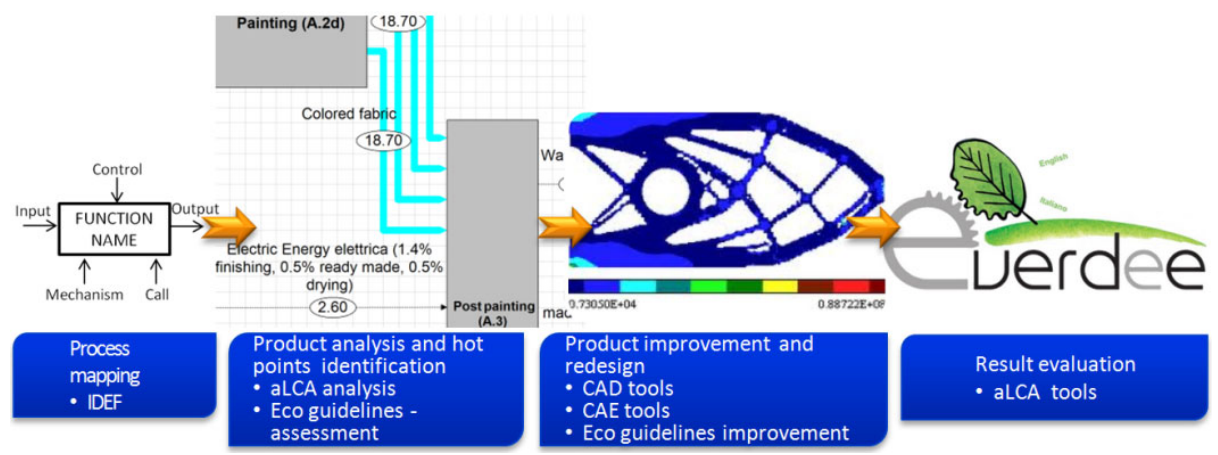

Fig. 1. Tools combination for the proposed ECO-desing approach

The overall process, schematized in figure 2, consists in mapping the process by a modified IDEF0 diagram. Then a software interface translates IDEF data for a simplified LCA software developed by ENEA, called EverdEE software. Once environmental impacts are calculated, a list of hotspots are generated in order to define a list of directions to intervene. Such a list is conceived according to the most promising intervention that can be provided for more sustainable process/product instead of generating the list of items associated with the maximum impact. Improuving phases are supported by a set of Eco-guidelines, presented in the following section. Results are finally compared by eVerdEE tool to provide a quantitative estimate of ECO- impacts. Once the overall process will be fully integrate in a single modeling tool ( CAD+ Eco-guidelines+aLCA+ structural optimization sw), it will be possible to suggest users in real time if any geometric modification is going in the right eco-directions and automatically provide a better geometry.

\section{REMAKE ECO-Guidelines}

REMAKE guidelines being extracted from the TRIZ laws of evolution [30], aiming to reduce resource consumption (mainly material and energy) increasing efficiency. This is possible by taking into account the best heuristics and theories of problem solving, and also taking into account new trends, technologies and best practices in green design.

REMAKE-guidelines allow guiding the designer in order to improve the environmental performance of the virtual product. They actually constitute over 330 actions at user level, organized by pre-manufacturing, manufacturing, product use and end of life.

Each phase contains a set of objects to which the guidelines refer to. For every object a list of goals to be achieved, opportunely translated in terms of resource abatement, is provided in order to design a better product.

A selection of such guidelines has been selected to be integrated as supporting knowledge for an ECO-Design activity based on CAD modeling; in fact they involve the entire life-cycle. 
Such suggestions have been adapted to design in order to maximize structural optimizers potentialities, guiding the user to design a less impact product (e.g. working on chemical composition of raw materials, making manufacturing process easier, a lighter and more compact product during the functioning, energetically more effective, easier to transport, disassembly, dispose, etc.).

In this work, the authors show the guidelines related to the material minimization and volume wastage, saving performances and avoiding the complexity of manufacturing.

At differences of other ECO-improvement tools, the peculiarity of this approach is that the designer, following the suggestions directly proposed inside the CAD environment, can visualize in real-time not only how the current phase changes but also the impacts on all the other phases belonging to the whole product life-cycle, thus allowing 360 degree changes.

The results are obtained using the eVerdEE, an aLCA software developed by Enea. This software allows to assess the impacts on a large number of eco-sustainability indicators. It contains well updated libraries and it can be easy interface to information flow moving from a CAD based process.

REMAKE guidelines are organized in 3 levels:

- guidelines with the aim to minimize the target resource;

- guidelines guiding the user on which CAE tool has to be chosen and eventually the proper information library;

- guidelines suggesting the specific strategy to better achieve the desired results.

For some strategies a more detailed path has been developed. Just to clarify, few examples, one for phase, are shown in the following:

In table 1 an example about pre-manufacturing phase, and for use phase is given. The goal is to reduce the impacts, working on product supply phase from cradle to gate, re-setting the geometry of the given piece and of its packaging in order to minimize mass, volume and impact on transports and inner logistics of the materials entering in the factory.

Table 1. One example taken from a list of strategies for the Pre-manufacturing phase, and for the Use phase

\begin{tabular}{|c|c|c|}
\hline Goal & Tools & CAE strategy \\
\hline $\begin{array}{c}\text { Minimize } \\
\text { Volume and/or } \\
\text { Mass wastage of } \\
\text { raw material }\end{array}$ & $\begin{array}{c}\text { CAD modeling } \\
+\end{array}$ & $\begin{array}{c}\text { Defining the minimum working volume, } \\
\text { based on the maximum dimensions of raw } \\
\text { material from which the component has to be } \\
\text { extracted - set the optimization strategy } \\
\text { (topological or free size) constraint and the } \\
\text { path }\end{array}$ \\
$\begin{array}{c}\text { specific constraints of minimum member size } \\
\text { (eventually combine with draw direction and } \\
\text { symmetry). }\end{array}$ \\
\hline
\end{tabular}

For the sake of brevity we omit the MSTP strategies (Modal Shift Transportation Planner)[20] for optimizing the transports of goods and the PPP tools (Production Process Planning) to better manage the flow and the replenishment of material and machining wastes. 
Table 2. One example taken from a list of strategies for the Phase of Manufacturing

\begin{tabular}{|c|c|c|}
\hline Goal & Tools & CAE strategy \\
\hline \multirow{4}{*}{$\begin{array}{c}\text { Manufacturing } \\
\text { Energy and Mass } \\
\text { reduction }\end{array}$} & $\begin{array}{c}\text { Thermo mechanics } \\
\text { coupling } \\
\text { optimization }\end{array}$ & $\begin{array}{c}\text { Simulation and maximization of the energy- } \\
\text { saving of the tool path during CNC } \\
\text { machining }\end{array}$ \\
\cline { 2 - 3 } & $\begin{array}{c}\text { Thermo-mechanical coupling calculation is } \\
\text { used for improving optimization performance } \\
\text { in order to maximize the Energy-saving and } \\
\text { material use during foundry process }\end{array}$ \\
\cline { 2 - 3 } & $\begin{array}{c}\text { Set up the geometry of finished work-piece } \\
\text { osing a structural optimizer and a proper } \\
\text { material }\end{array}$ \\
\hline
\end{tabular}

This strategy is based on the assumption that a more compact rough machining impacts in a positive way on all the following phases. So, in this process phase the designer task is to identify the proper shape of the component in order to achieve the desired performance using the minimum material.

The REMAKE guidelines support step-by-step the designer during the creation of different shapes, such shapes are put inside CAD, where they can be evaluated by eVerdEE. In particular, for each shape eVerdEE calculates the global impact on the entire life-cycle.

The result is a series of scenarios comparing the different design choices. In this specific case, the designer will be able to understand if it is better to:

- maximize the raw piece volume as much as possible and working on the minimization of the finished work-piece mass in order to improve the use phase, or potentially the manufacturing phase.

- minimize the raw piece volume as much as possible in order to have greater benefits during pre-manufacturing phase.

In this work the authors focus their attention on the potentialities of topological optimization tools, even though the current guidelines (approximately 20) concern also other different virtualization tools to support the eco-sustainable design activity in all phases, such as:

- CAM, used for instance to simulate and maximize the energy-saving of the tool path during CNC machining.

- Thermal optimization to improve the impacts on foundry processes.

- Multidisciplinary optimization to combine optimization strategies and innovative material choice.

- Computer aided assembly and disassembly for manufacturing and end of use phases.

- Production process planning.

- Modal shift transportation planner.

- Vehicle routing planner to better manage the logistic of material handling, storage, etc.

The following picture shows the integrated ECO-design process. A deeper analysis on how REMAKE Guidelines can be integrated in it is given in the work of Russo [21]. 


\section{Design Optimization}

Designing by optimization techniques means translating a design task into a mathematical problem with the following basic entities:

- An objective function, i.e. the performance of the system that the designer wants to reach or to improve.

- A set of design variables, i.e. the parameters of the system affecting the objective function.

- A set of loading conditions and constraints representing the requirements the system has to satisfy.

The optimization algorithm finds the value of the design variables which minimizes, maximizes, or, in general, "improves" the objective function while satisfying the constraints.

The use of computers for design optimization is rather common in several fields since 1980's; besides, during the last years new optimization tools have been developed to solve specific design problems [4]. In the followings the main features of these techniques will be summarized. All techniques can be set up with the aim of reducing mass as least as possible.

In a shape optimization process the outer boundary of the structure is modified according to the optimization task. The shape of the structure, modeled through the finite element method, is modified by the node locations: the optimization algorithm, according to the loads and boundary conditions applied to the FE model, changes the coordinates of the nodes which are defined as design variables. The result of the optimization cycle is a deformed geometry of the starting shape structure.

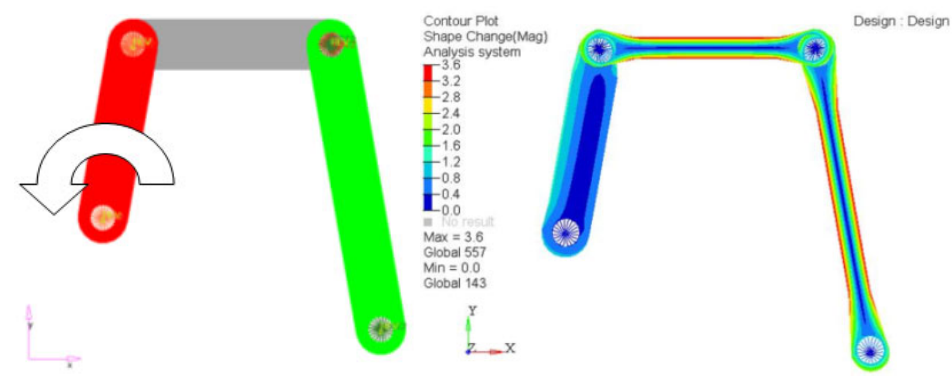

Fig. 2. An example taken from Optistruct tutorials 2009, regarding a mass reduction for a bar linkage obtained by a shape optimization

The size optimization, is a special type of parametrical optimization in which the design variables are represented by some properties of structural elements such as shell thickness, beam cross-sectional properties, spring stiffness, mass, etc. During the optimization process these parameters are modified by the algorithm until the expected goal is reached.

Topology optimization is a technique that determines the optimal material distribution within a given design space, by modifying the apparent material density 
considered as a design variable. The design domain is subdivided into finite elements and the optimization algorithm alters the material distribution within the design space at each iteration, according to the objective and constraints defined by the user. The external surfaces, defined as "functional" by the user, are kept out from the optimization process and considered as "frozen" areas by the algorithm.
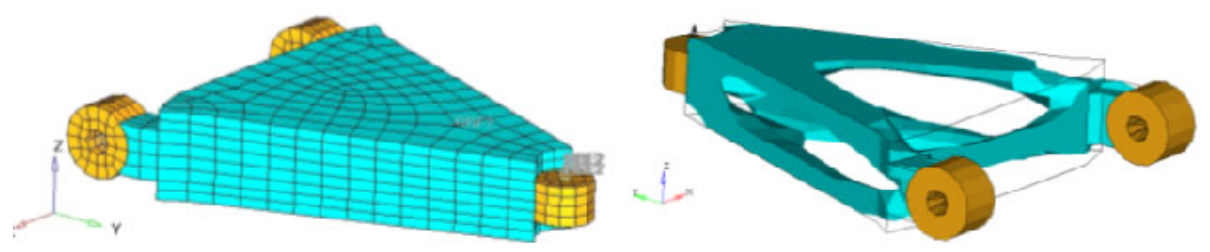

Fig. 3. An example taken from Optistruct tutorials 2009, regarding a mass reduction for a control arm obtained by a topological optimization

Topography optimization is an advanced form of shape optimization in which a distribution of ribs and pattern reinforcements are generated on a specific design region. The approach in topography optimization is similar to the approach used in topology optimization, but shape variables (node coordinates) are used instead of density variables. The large number of shape variables allows the user to create any reinforcement pattern within the design domain.

Moreover manufacturing constraints may be set in order to take into account the requirements related to the manufacturing process. Sliding planes and preferred draw directions may be imposed for molded, tooled and stamped parts as well as minimum or maximum size of the structural elements (i.e. ribs, wall thicknesses, etc.).

From an eco-design point of view, such constraints play a strategic role to obtain the final geometry, that achieves the objectives of mass and volume minimization without increasing the complexity in the manufacturing processes.
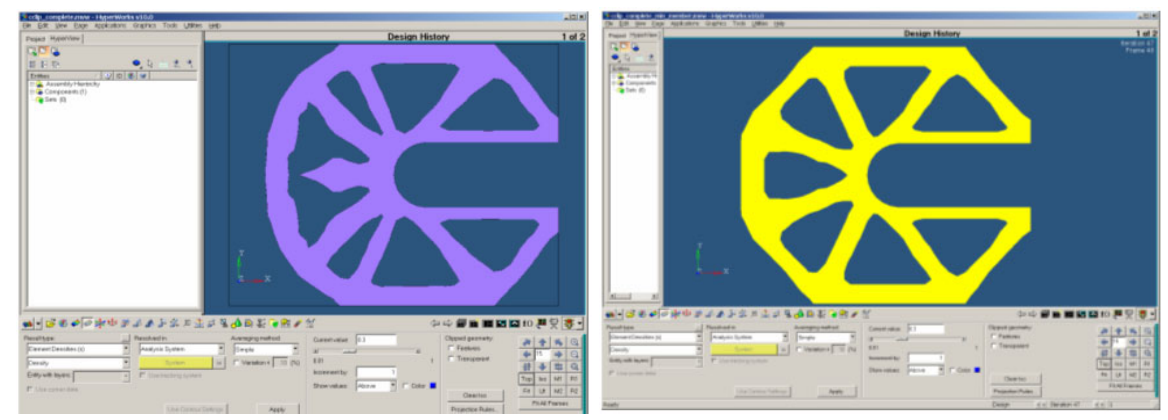

Fig. 4. Comparing between two optimizations. On the right the same piece with minimum member size constraints. Notice the smaller members in the original iso surface plot are replaced by a more discrete rib pattern. The second design is so easier to manufacture.

So, most of optimizers functionalities are already well integrated with an Ecodesign view, nevertheless it is necessary to use a network of guidelines in order to 
better control all the design choices and consider these choices influence the other phases of the product life-cycle. As we said before, the network of guidelines shows some preferred path for combining optimizations between strategies and constraints, and at the same time it gives information about how a choice influence the environmental indicators directly in the design phase and not in the next phases as using validation tools.

\section{Case Study}

The following case study shows how the guidelines and structural optimization tools can be combined in order to obtain more sustainable products.

In particular, the application of the methodology concerns a steering plate mounted by the Spanish Producer Gas Gas on a wide range of trial motorcycles. The mechanical properties are the same as the ones mounted on the other motorcycles types, but one fundamental requirement of the trial motorcycles plate is the lightweight.

The plate is produced by die-casting process and the production rate is 10.000 units per year. Therefore, this value has been used as a functional unit for preliminary calculation by eVerdEE.

The used guidelines are the same explained in section 4: mass minimization during the use phase and minimization of raw material volume during the pre-manufacturing phase. For simplicity, others guidelines related to the manufacturing phase are not take into account in this application.

The guidelines regarding to the use phase (topological optimization combined with the constraint of minimum member size, with or without the draw direction constraint) generate 2 different geometric scenarios.

In case of guidelines related to the pre-manufacturing phase, the suggested procedure starts with a modification of the initial volume of the raw piece (in this specific case the volume reduction was fixed arbitrarily at $-15 \%)$, then the next step is a topological optimization choice (or free size optimization) combined with the same constraints explained for the use phase. So, from the previous step, 4 different geometries are obtained as shown in table 4.

The 4 geometries have been evaluated according to sustainability requirements, considering 2 typologies of production: die-casting process and CNC machining tools. Data, such as working energies, time and material wastes, have been obtained by simulations performed by experts. While, the impacts on transports, packaging and end of life have not been considered, because they are approximated as invariant and irrelevant for the geometries valuated.

Finally, 8 different scenarios were produced (the 4 models realized by die-casting process and the same 4 models calculated using CNC machining tools). Calculation was produced by adopting eVerdEE software. In figure 7 , graphs related to environmental impact analysis of the 8 models are shown. The graphs show, for each model, the reduction in percentage of $\mathrm{CO} 2 \mathrm{eq}$ production and the mass reduction in percentage. 
Table 3. Alternative plate configurations

\begin{tabular}{|l|c|c|}
\hline & $\begin{array}{c}\text { USE PHASE GUID. } \\
\rightarrow \text { Reduce the mass of } \\
\text { finite product }\end{array}$ & $\begin{array}{c}\text { PRE-MANUF. GUID. } \\
\rightarrow \text { Reduce the volume } \\
\text { of raw material }\end{array}$ \\
\hline Initial product & $\begin{array}{c}\text { Topological optimization: } \\
\text { Minimum member size }\end{array}$ & $\begin{array}{c}\text { Topological optimization: } \\
\text { Minimum member size }\end{array}$ \\
\hline Topological optimization: \\
Minimum member size \\
Draw direction
\end{tabular}

The first comparison emerging from the graph is that, for the batch of production considered, the $\mathrm{CO} 2 \mathrm{eq}$ generated by machining is higher than the one generate by diecasting. This difference decreases as the batch decreases.

In case of machining, the best result, in terms of CO2eq, is obtained by the $4^{\text {th }}$ configuration (coming from pre-manufacturing guidelines and adopting the draw direction constraint). This model, compared with the original one, allows a $15 \%$ of reduction of $\mathrm{CO} 2 \mathrm{eq}$ (equal to $3 \mathrm{Kg}$ of $\mathrm{CO} 2 \mathrm{eq}$ ). Such a reduction is due to 2 main reasons:

1. The application of pre-manufacturing guidelines, concerning the mass and raw piece reduction. These 2 directions of intervention have an high positive impact on the CNC machining tools.

2. The simultaneous imposition of constrains (minimum member size and draw direction). They influence the manufacturing stage through an high simplification of the finished piece geometry (allowing at the same time a mass reduction).

For machining calculation, the gain obtained, in pre-manufacturing and manufacturing phase, by the $4^{\text {th }}$ configuration is so high that it is convenient even though this variant is heavier than the others. In this case a design strategy aiming at minimizing energy and mass in the phase of use would not have been the right direction.

Also the $3^{\text {rd }}$ configuration gives an high $\mathrm{CO} 2 \mathrm{eq}$ reduction, obtained by the volume reduction of the raw piece, but some of the benefits obtained during the premanufacturing are lost during the manufacturing. In fact the draw direction constrain has not been set up and so the plate geometry is more complex, this optimization negatively influences the manufacturing. 

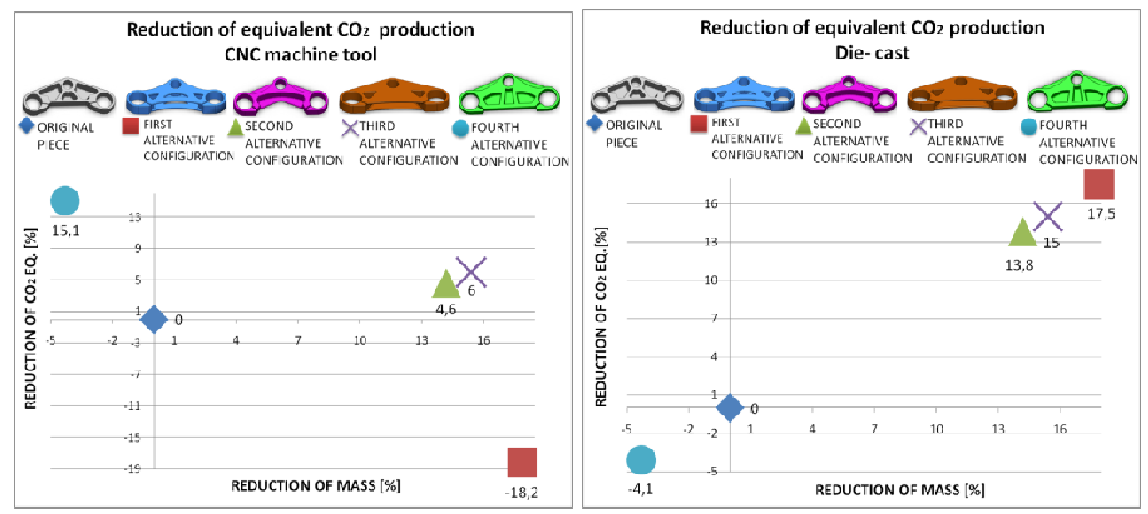

Fig. 5. Graph shows final results of the ecodesign process. Resulting LCA scenarios are calculated in terms of $\mathrm{CO}_{2}$ eq reduction.

In this respect, we can compare the $4^{\text {th }}$ and the $1^{\text {st }}$ configuration. Using the $1^{\text {st }}$ configuration a mass reduction of about $20 \%$ can be achieved, but the high resources consumption in the manufacturing eliminates all the benefits gained during the use.

Considering the models related to the die-casting, the best result is obtained by the $1^{\text {st }}$ configuration, that allows a reduction of $\mathrm{CO} 2 \mathrm{eq}$ of $17 \%$. This benefit mainly derived from the application of the guideline related to the use, that focus on a mass reduction.

This guideline, for the die-casting process, has an high positive impact also on the manufacturing, because it permits a less resources exploitation (in opposition to the machining case).

The $4^{\text {th }}$ configuration is the worst result from the eco-sustainability point of view. Such a configuration produces a quantity of $\mathrm{CO} 2 \mathrm{eq}$ much higher than the $1^{\text {st }}$ one, because it has a higher mass negatively impacting on the use and manufacturing. Thus, it is possible to state that, at difference of CNC machining tools, for die-casting process the imposition of the 2 constrains (minimum member size and draw direction) has poor influence on the sustainability.

It 's obvious that it is difficult for the designer to control all the implications of its choices when the goal is extended to the entire lifecycle of the product.

This integrated system of tools allows user to create multiple not conventional scenarios that clearly indicate which is the best route to take.

By a further sensitivity analysis conducted on each variant, the designer can decide which is the best compromise between the results obtained, considering performance and eco-sustainability requirements.

\section{Conclusions}

This work lays the foundations for an eco-design path integrating CAE tools with LCA aproach. This path includes different approaches for allowing designers to get useful information in order to create a more sustainable product directly in CAE environment. In particular, it has been shown how the introduction of a structural optimization tool into the ECOdesign process is a very useful tool, guiding also not expert designer to manage complex shapes and not conventional geometries. In order 
to manage them, eco-sustainability criteria have been opportunely interpreted for fixing objectives and constraints.

The peculiarity of the method proposed is that the modifications of the pieces are evaluated directly on the entire product life cycle. The case study has demonstrated how can be difficult for a designer control and manage all implications. The methodology helps designer to easily visualize the best direction, By means of guidelines, a set of design alternatives can be created and, representing them on a graphic, they can be easily evaluated. Once the best configuration is fixed, a deeper optimization cycle can be provided to the initial product following direction suggested by the method in order to achieve the maximum reachable benefit .

At the moment, the author are developing precise procedures for the application of the entire set of guidelines involving structural optimization tools and more in general others CAE tools. In particular the benefits reachable by adopting multi cycle optimization and multi-body optimization are under evaluation.

\section{Acknowledgments}

The author would like to thank Marco Serafini and Valentino Birolini from University of Bergamo for their contribution to the development of the present research. The authors sincerely thanks Fondazione Cariplo for partially funding the researches that lead to this paper.

\section{References}

1. Knight, W., Curtis, M.: Design for environment software development. Journal of Sustainable Product Design 9, 36-44 (1999)

2. Van Nes, N., Cramer, J.: Design strategies for the lifetime optimisation of products. Journal of Sustainable Product Design 3, 101-107 (2003)

3. Lofthouse, V., Bhamra, T.: End of life decisions - a design guide. In: 11th Annual International Sustainable Development Research Conference. University of Tampere, Helsinki (2005)

4. Herrmann, C., Frad, A., Luger, T., Krause, F.L., Ragan, Z.: Integrating end-of-life evaluation in conceptual design. In: IEEE International Symposium on Electronics and the Environment, pp. 245-250 (2006)

5. Clark, T., Adams, G.: Smart ecodesign: eco-design checklist. For Electronic Manufacturers, 'Systems Integrators', and Suppliers of Components and Subassemblies. Version 2. Centre for Sustainable Design, Farnham (2002)

6. ECMA. Environmental design considerations for ICT \& CE products. Standard: ECMA-341, 2nd ed. ECMA-International, Geneva (2004)

7. Smiths: Eco-design guide. Smiths Group internal document (1) (2004)

8. Tischner, U., Dietz, B.: The toolbox: useful tools for ecodesign. In: German Federal Environmental Agency (ed.) How to do ecodesign. A Guide for Environmentally and Economically Sound Design. Verlag form GmbH, Frankfurt (2000)

9. Persson, J.G.: Eco-indicators in product development. Institution of Mechanical Engineers, Part B: Journal of Engineering Manufacture 215(5), 627-635 (2001)

10. Tingstrom, J., Karlsson, R.: The relationship between environmental analyses and the dialogue process in product development. Journal of Cleaner Production 14(15-16), 1409-1419 (2006) 
11. Fuller, K.: Environmental impact assessment. In: Brady, J. (ed.) Environmental Management in Organisations. The IEMA Handbook. Earthscan, London (2005)

12. Boyd, S., Dornfeld, D., Krishnan, N.: Life cycle inventory of a CMOS Chip. In: IEEE International Symposium on Electronics and the Environment, pp. 253-257 (2006)

13. BSI. BS EN ISO14040:2006, Environmental management - life cycle assessment principles and framework. British Standards Institution, London (2006)

14. Kobayashi, H.: Strategic evolution of eco-products: a product life cycle planning methodology. Research in Engineering Design 16(1-2), 1-16 (2005)

15. Bhamra, T.A., Evans, S.: An ecodesign model based on industry experience. In: IEE Seminar on: Engineering and the Environment - How it Affects You!, London, pp. 8/1-8/4 (1999)

16. Knight, P., Jenkins, J.O.: Adopting and applying eco-design techniques: a practitioners perspective. Journal of Cleaner Production 17, 549-555 (2009)

17. Russo, D., Regazzoni, D.: TRIZ Laws of evolution as eco-innovation method. In: IDMME - Virtual Concept 2008, Beijing, China (2008)

18. Todd, J.E., Curran, M.A.: Streamlined life-cycle assessment: a final report from the SETAC North America Streamlined LCA Workgroup. SETAC (1999)

19. Yezersky, G.: General Theory of Innovation. An Overview. In: Trends in Computer Aided Innovation. IFIP International Federation for Information Processing, vol. 250 (2007)

20. Amano, M., Yoshizumi, T., Okano, H.: The modal-shift transportation planning problem and its fast steepest descent algorithm. In: Proceedings of the Simulation Conference 2003, vol. 2, pp. 1720-1728 (2003)

21. Russo, D., Bersano, G., Birolini, V., Uhl, R.: European testing of the efficiency of TRIZ in eco-innovation projects for manufacturing SMEs. In: TRIZ Future Conference 2010, Bergamo Italy (2010)

22. James, P.: The Sustainability Circle: a new tool for product development and design. Journal of Sustainable Product Design (2), 52-57 (1997)

23. Bruntland, G.H.: Report of the World Commission on Environment and Development (WCED), Our Common Future (1987); Oxford University Press, Oxford (1997)

24. Tri-Ngoc, P.V., Reiner, A.: Spreadsheet-Based Models With Object-Oriented Approach: Development of Sustainable Products. In: 24th Computers and Information in Engineering Conference, Salt Lake City, Utah, USA, pp. 61-67 (2004)

25. Leibrecht, S.: Fundamental principles for CAD-based ecological assessments. International Journal of Life Cycle Assessment 10(6), 436-444 (2005)

26. Cappelli, F., Delogu, M., Pierini, M.: Integration of LCA and EcoDesign guideline in a virtual cad framework. In: 13th CIRP International Conference on Life Cycle Engineering, Leuven (2006)

27. Wolfgang, W., Rainer, Z., Kun-Mo, L.: ECODESIGN implementation: a systematic guidance on integrating environmental considerations into product development. Springer, Heidelberg (2004)

28. Willems, B., Dewulf, W., Duflou, J.R.: Active snap-fit development using topology optimization. International Journal of Production Research 45, 4163-4187 (2007)

29. Recycling and Resource Efficiency driving innovation in European Manufacturing SMEs, REMake (2010),

http: / /www. europe-innova.eu/web/guest/eco-innovation/

eco-innovation-platform/remake/about

30. Altshuller, G.S.: Creativity as an exact science: the theory of the solution of inventive problems. Gordon and Breach Science Publishers, New York (1984) 